\title{
Reabilitação do sorriso por meio da utilização de prótese total imediata: relato de caso
}

\author{
Rehabilitation of smile through immediate total prosthesis: case report
}

Rehabilitación de la sonrisa a través de la prótesis total inmediata: caso clínico

Daniel Sartorelli Marques de Castro $^{1 *}$, lana Aragão Magalhães ${ }^{1}$, Brenna Alves de Lima ${ }^{1}$, Hilmo Barreto Leite Falcão Filho², Jozely Francisca Mello Lima², Francisco Nathizael Ribeiro Gonçalves ${ }^{1}$, George Táccio de Miranda Candeiro'.

\section{RESUMO}

Objetivo: Relatar a utilização das próteses totais imediatas na obtenção da estética e da função nos casos de reabilitação oral. Relato de experiência: Paciente A.B.S, 43 anos de idade, apresentou, como queixa principal, mobilidade dentária e insatisfação com seu sorriso. Ao exame clínico inicial, observou-se a presença de poucos elementos dentários, presença de mobilidade grau III, cálculo e sangramento gengival. Foi realizado todo o planejamento protético prévio e as exodontias foram realizadas e, concomitantemente, foi feita uma regularização do rebordo, visando a uma melhor adaptação da base da prótese. O controle pósoperatório foi realizado após $07,21,30$ e 64 dias, e no último controle, foi iniciada a confecção da prótese definitiva. Considerações finais: $O$ prognóstico clínico favorável dessa modalidade de reabilitação dependerá da proservação de cada caso, com visitas periódicas para ajustes que possam ser necessários e colaboração do paciente. Esses aspectos determinarão o prognóstico favorável e a longevidade do trabalho protético executado.

Palavras Chaves: Prótese total, Prótese total imediata, Estética dentária.

\begin{abstract}
Objective: to report the use of immediate total prostheses in obtaining aesthetics and function. Experience Report: Patient A.B.S., 43 years of age, presented, as main complaint, dental mobility and dissatisfaction with his smile. The initial clinical examination revealed the presence of few dental elements, the presence of grade III mobility, gingival calculus and bleeding. All prosthetic planning was previously carried out and the exodontia was performed, concomitantly, a regularization of the ridge was performed, aiming at a better adaptation of the prosthesis base. The postoperative control was performed after $7,21,30$ and 64 days, and in the last control, the production of the definitive prosthesis was started. Final Considerations: The favorable clinical prognosis of this type of rehabilitation will depend on the continuation of each case, with periodic visits for adjustments that may be necessary and patient collaboration. These aspects will determine the favorable prognosis and longevity of the prosthetic work performed.
\end{abstract}

Key words: Complete denture, Immediate complete denture, Dental esthetics.

\section{RESUMEN}

Objetivo: Informar el uso de prótesis completas inmediatas para obtener estética y función en casos de rehabilitación oral. Informe de experiencia: el paciente A.B.S, de 43 años de edad, presentó, como queja principal, movilidad dental e insatisfacción con su sonrisa. En el examen clínico inicial, se observaron pocos

${ }^{1}$ Universidade Christus (UNICHRISTUS), Fortaleza - CE. *E-mail: danielsartorelli@gmail.com

2 Universidade Federal do Ceará (UFC), Sobral - CE. 
elementos dentales, movilidad de grado III, cálculo y sangrado gingival. Se llevó a cabo toda la planificación protésica previa y se realizaron extracciones y, al mismo tiempo, se regularizó el borde, con el objetivo de una mejor adaptación de la base de la prótesis. El control postoperatorio se realizó después de 07, 21, 30 y 64 días, y en el último control se inició la prótesis definitiva. Consideraciones finales: El pronóstico clínico favorable de este tipo de rehabilitación dependerá de la continuación de cada caso, con visitas periódicas para ajustes que puedan ser necesarios y la colaboración del paciente. Estos aspectos determinarán el pronóstico favorable y la longevidad del trabajo protésico realizado.

Palabras Clave: Dentadura completa, Dentadura completa inmediata, Estética dental.

\section{INTRODUÇÃO}

A perda dos dentes é uma condição que leva a alterações fisiológicas, sociais, nutricionais e psicológicas (DE MEDEIROS JJ, et al., 2012; EMAMI E, et al., 2013). Apesar dos avanços na odontologia preventiva e restauradora, parte da população ainda sofre com esse mal (BÜTTEL AE, et al., 2012; ST GEORGE GS, et al., 2010). Estudos mostram que a perda dentária tem como principais causas a cárie, a doença periodontal e o traumatismo (BÜTTEL AE, et al., 2012 ), podendo-se associar a múltiplas comorbidades sistêmicas, como obesidade, eventos relacionados à doença pulmonar obstrutiva crônica (DPOC), pneumonia, câncer de cabeça e pescoço e declínio cognitivo (FELTON DA, 2015; POLZER I, et al., 2012).

Diante da perda parcial ou total dos dentes, os profissionais podem aderir a modalidades reabilitadoras que incluam próteses fixas ou removíveis, sejam elas parciais, sejam totais (PRESTON AJ, 2012). O sucesso das diferentes formas de reabilitação protética é influenciado por diversos fatores, como o acesso a serviços odontológicos, as condições locais e a capacidade de adaptação do paciente (JABLONSKI RY e BARBER MW, 2015). O tipo de modalidade reabilitadora a ser utilizada para cada paciente será determinada após uma anamnese e um exame clínico criterioso, associado à avaliação de exames complementares, como radiografias e tomografias computadorizadas, não negligenciando a viabilidade financeira do paciente (JOGEZAI U, et al., 2018).

Entre as modalidades utilizadas na reabilitação desses pacientes, a prótese total imediata (PTI) está indicada naqueles casos em que a condição dos dentes remanescentes não permita a confecção de nenhum outro tipo de reabilitação, devido ao envolvimento periodontal, às lesões cariosas, à distribuição inadequada dos pilares remanescentes ou às limitações financeiras (GOIATO MC, et al., 2013). A PTI será responsável por devolver os dentes ausentes, os tecidos moles e duros adjacentes, sendo uma maneira eficaz de manter a estética e a função do paciente durante a mudança de uma dentição natural para uma artificial (PRESTON AJ, 2012; ZARB GA, et al., 2013; ZITZMANN NU e MARINELLO CP, 2002).

As contraindicações para essa modalidade reabilitadora contemplam idades avançadas, comprometimentos sistêmicos descompensados, que trazem risco no trans e pós-operatório, pacientes pósradioterapia de cabeça e pescoço, não colaboradores e condições bucais que levem a grandes alterações no rebordo alveolar, não possibilitando a reabilitação imediata (GAVAZZONI A, et al., 2015).

Baseado nas condições bucais iniciais apresentadas pela paciente, tais como, a presença de poucos elementos dentários, doença periodontal e insatisfação estética com seu sorriso, o objetivo do presente artigo é, relatar o passo a passo clínico de uma reabilitação oral através do uso de uma prótese total imediata superior, ressaltando a importância do planejamento estético e funcional do tratamento reabilitador, assim como os cuidados em cada etapa do tratamento clínico, permitindo que o clínico possa obter resultados satisfatórios, mesmo nos casos mais complexos.

\section{RELATO DE CASO}

Paciente A.B.S, do sexo feminino e de 43 anos de idade procurou atendimento na Clínica odontológica, com a queixa principal de mobilidade dentária e insatisfação com seu sorriso. Ao exame clínico inicial, observou-se a presença de poucos elementos dentários em estado insatisfatório, tanto em maxila como em 
mandíbula. Foi evidenciada a presença de mobilidade grau III, a presença de cálculo e sangramento gengival nos remanescentes da arcada superior (elementos 16, 14, 13, 11, 21, 22, 23, 28), caracterizando uma doença periodontal severa (Figura 1).

Figura 1 - Imagens intra e extraorais, mostrando a condição clínica dos dentes superiores.

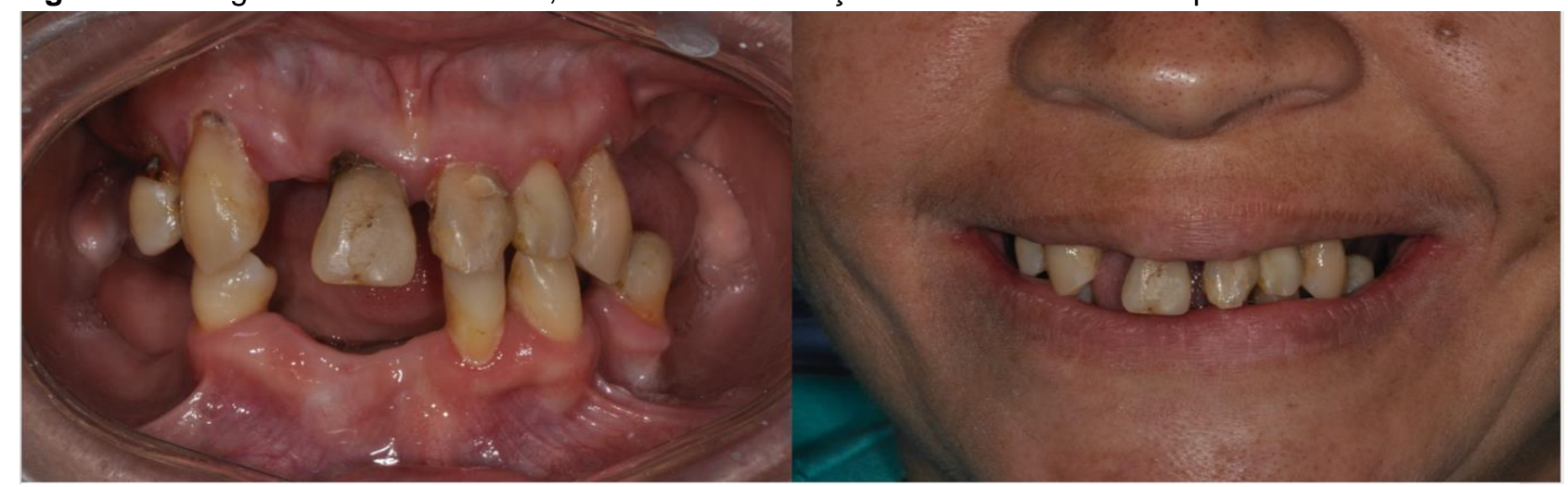

Fonte: Castro DSM, et al., 2020.

Diante dessas condições, a opção terapêutica foi proposta: explanou-se que seria necessária uma adequação da cavidade bucal prévia a essa reabilitação, a qual envolvia exodontias de todos os elementos da arcada superior, que seria reabilitada com prótese total imediata, e tratamento restaurador na arcada inferior, somado a confecção de prótese parcial removível. Durante a anamnese, a paciente não relatou nenhuma informação digna de nota ou que pudesse inviabilizar o procedimento cirúrgico. Foi realizada moldagem inicial para a individualização da moldeira nas áreas desdentadas, e, em seguida, realizada moldagem propriamente dita.

Para a adequação do meio bucal prévia ao procedimento para a instalação da prótese total imediata, foi realizada raspagem supragengival de todos os sextantes, exodontias dos elementos 16, 18 e 28 e procedimentos restauradores dos remanescentes mandibulares.

A moldagem inicial foi realizada com material elastomérico rígido (Silicone de condensação pesado Perfil/Vigodent S/A Indústria e Comércio) para a individualização da moldeira nas áreas desdentadas e, posteriormente, foi realizada a moldagem propriamente dita utilizando-se um hidrocoloide irreversível (Hidrogum 5, Zhermack Dental / Labordental Ltda). Depois do vazamento dos moldes, foram confeccionadas chapas de prova e planos de orientação para a realização de registro de mordida. A posição de trabalho escolhida para o caso foi a relação central, devido à perda de referências oclusais da paciente. Os modelos foram enviados para o laboratório para a realização de montagem de dentes (Figura 2).

Figura 2 - Modelos de trabalho, com os registros e linhas de referência posicionados.

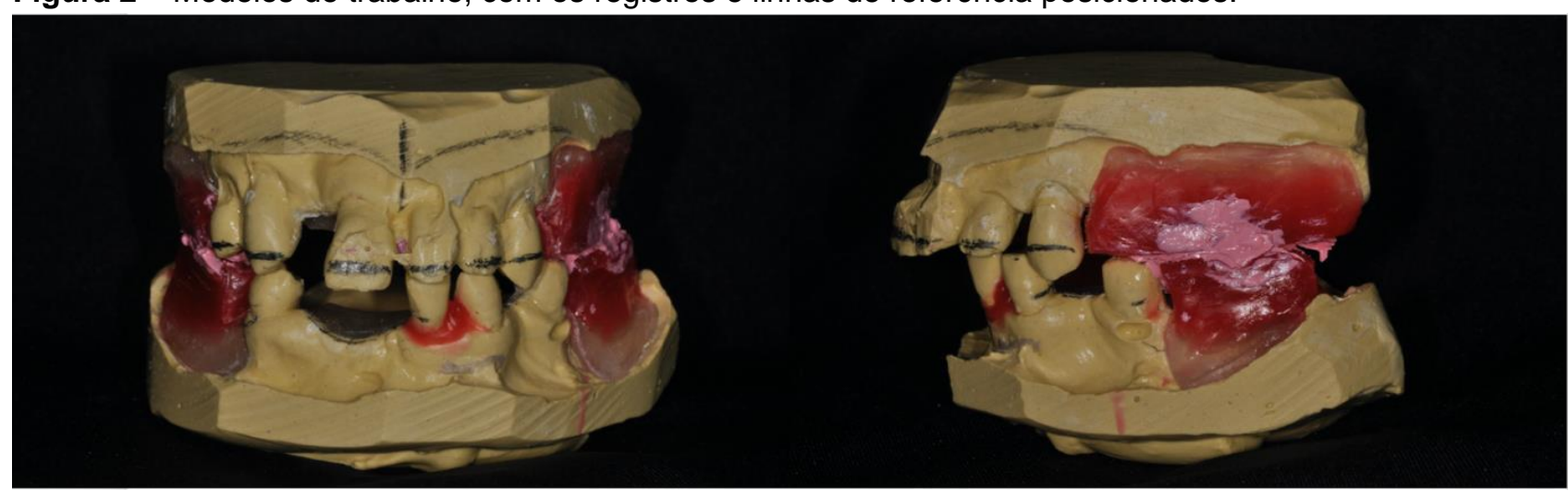

Fonte: Castro DSM et al., 2020. 
Nas exodontias dos elementos 14, 13, 11, 21, 22 e 23, foi realizado bloqueio bilateral do nervo alveolar superior anterior e nasopalatino, com uso de solução anestésica com vasoconstrictor (Mepivacaína $2 \%$ ). Diérese incisa foi realizada com lâmina número 15 ao redor dos colos dos elementos, seguida de diérese romba cautelosa com descolador de Molt e sindesmótomo, evitando a laceração dos tecidos. A cirurgia propriamente dita foi realizada com a técnica segunda, com o auxílio de fórceps número 150.

Posteriormente, foi realizada incisão interdental para ampliação do campo operatório e realização de regularização do rebordo, que foi feito com remoção de espículas com auxílio de alveolótomo, regularização grosseira com lima para osso, seguida da utilização de broca de tungstênio Maxicut, em caneta de baixa rotação, sendo válido ressaltar que a base da prótese foi utilizada como guia cirúrgico.

Sutura contínua festonada realizada com fio de seda 3-0 (PR - Procare) permitiu a coaptação passiva dos rebordos (sem tensão tecidual), visando a manter a forma delineada pela regularização do rebordo e permitindo a melhor adaptação da PTI (Figura 3).

Figura 3 - Rebordo superior após a extração e correção óssea e altura realizada.

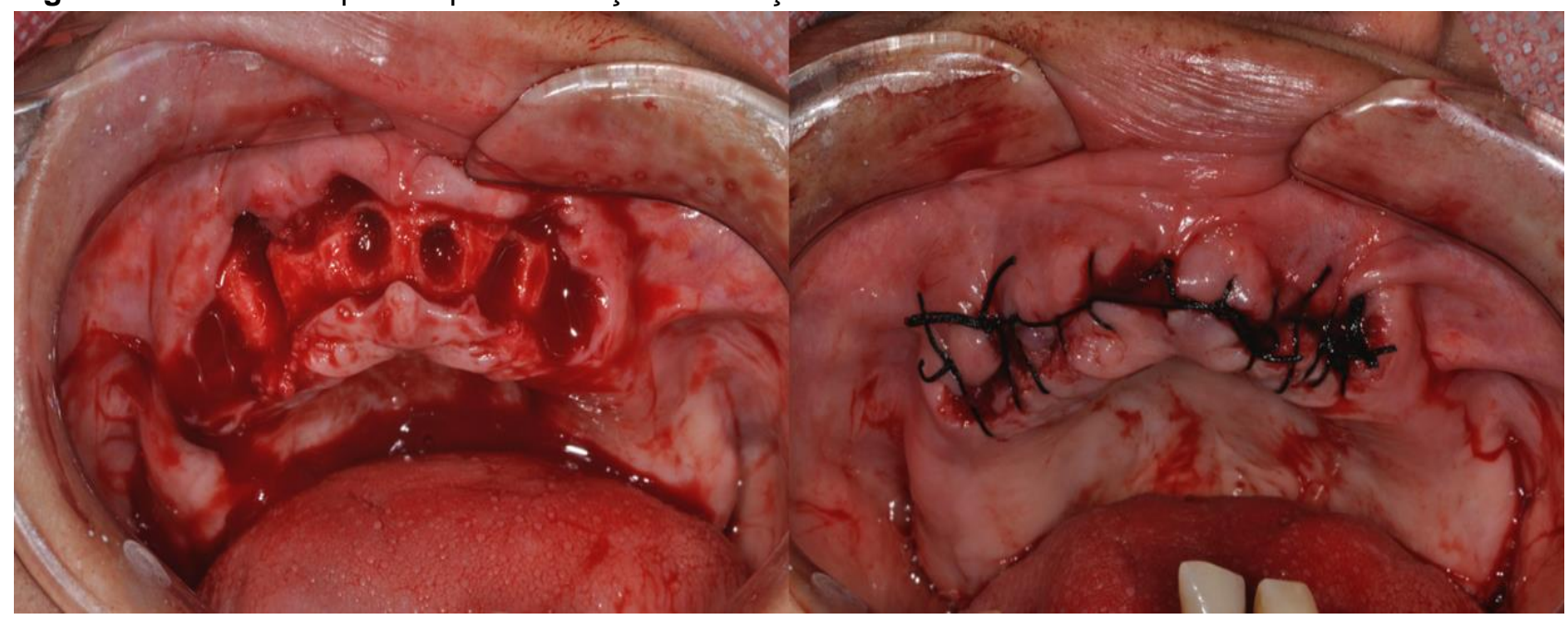

Fonte: Castro DSM et al., 2020.

Orientações pós-operatórias incluíram prescrição de antibiótico (azitromicina 500 mg, um comprimido por dia, durante três dias), anti-inflamatório (Nimesulida $100 \mathrm{mg}$, um comprimido de 12/12 horas, durante três dias) e analgésico (paracetamol $750 \mathrm{mg}$, de 8/8 horas, por 3 dias). Após a realização da sutura, a prótese total imediata foi ajustada para se adaptar ao rebordo alveolar superior com material soft (Softconfort / Dencril Produtos Odontológicos) (Figura 4).

Figura 4 - Prótese imediata sendo reembasada com um condicionador de tecido resiliente.

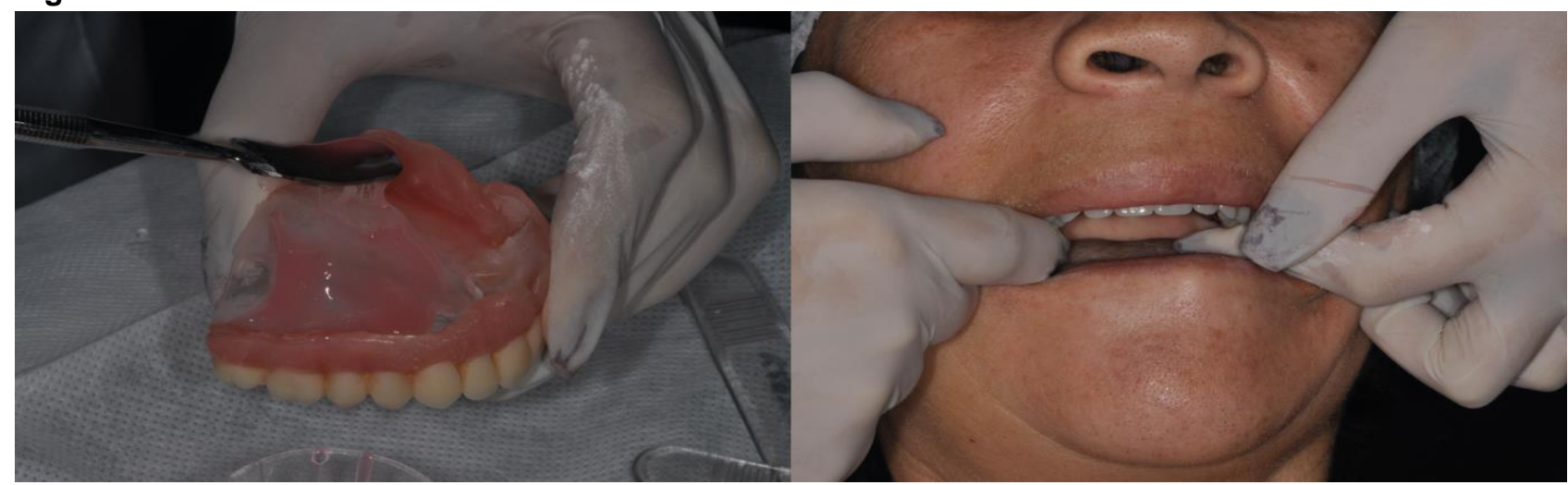

Fonte: Castro DSM et al., 2020. 
Ademais, a paciente foi orientada a não remover a prótese nas primeiras $24 \mathrm{~h}$, para permitir a estabilização do coágulo e a contenção do edema pós-operatório. Depois desse período, deve removê-la para higienização (Figura 5).

Figura 5 - Aspecto pós-operatório imediato após a instalação da prótese total.

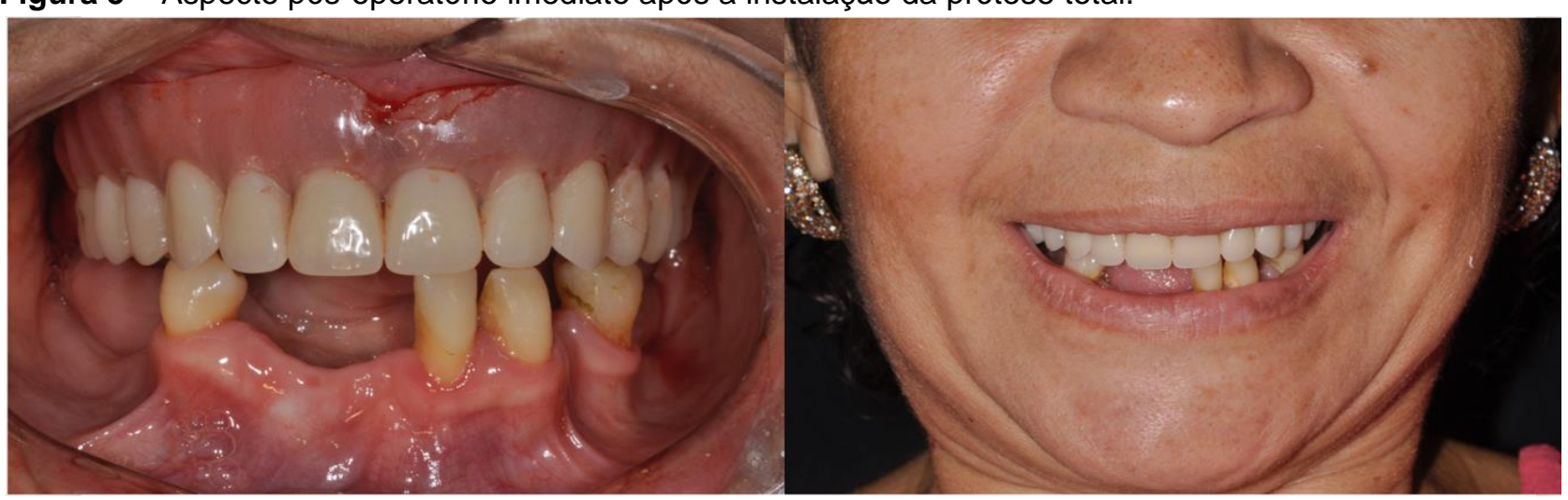

Fonte: Castro DSM et al., 2020.

O controle pós-operatório foi realizado após 7 e 21 dias, quando foi realizada a avaliação da ferida cirúrgica e a troca do condicionador de tecido soft (Softconfort / Dencril Produtos Odontológicos). O acompanhamento seguiu sendo realizado com 30 e 64 dias, período no qual a prótese provisória recebeu um reembasamento com material rígido (Cold Liner Reembase / TDV Dental LTDA). Durante o período pós-operatório, não houve nenhuma intercorrência, o processo de cicatrização ocorreu normalmente, permitindo que a prótese definitiva fosse realizada dentro do período programado.

\section{DISCUSSÃO}

Próteses dentárias são alternativas reabilitadoras que têm como objetivo restabelecer as funções do sistema estomatognático que incluem função mastigatória, fonação e estética. Essas alternativas podem ser mediatas ou imediatas, sendo definidas em face da conversa do profissional com o paciente acerca das vantagens e as desvantagens de cada terapêutica (MELETI VR, et al., 2002).

Essa modalidade de tratamento permite minimizar a transição entre o estado dentado para 0 estado desdentado do paciente. Esse aspecto auxilia o enfrentamento diante das questões psicossociais nas quais ele está inserido (PATEL J, et al., 2018). No presente caso, a paciente optou por essa modalidade devido à possibilidade de obtenção de estética e das características funcionais (oclusão e fonética) logo após o procedimento cirúrgico, outro aspecto importante, é a possibilidade de proporcionar um período mais tranquilo de adaptação em face da nova condição bucal (PATEL J, et al., 2018).

Suas indicações incluem casos em que os remanescentes dentários presentes em boca apresentem uma relação vertical apropriada, a qual permitirá a preservação das relações musculares mais próximas às originais, facilitando, assim, a manutenção dos aspectos funcionais como fala, mastigação, deglutição e aspectos estéticos (GOMES AC, et al., 2014; KRALJEVIE S, et al., 2001; TELLES D, 2009). Somado a isso, a presença desses remanescentes facilitará a seleção dos dentes artificiais, visto que o profissional terá parâmetros palpáveis como forma, tamanho e cor para serem levados em consideração (KRALJEVIE S, et al., 2001). Ademais, essa modalidade terapêutica permite menores taxas de hemorragias (KRALJEVIE S, et al., 2001) e reabsorções da crista óssea (JOHNSON K, 2966), hemostasia pós-operatória (PRESTON AJ, 2012) e previne a entrada de alimentos na ferida cirúrgica, levando à cicatrização mais rápida (PATEL J, et al., 2018).

Como desvantagem, pode-se ressaltar que pacientes sem a correta orientação acerca da complexidade dos procedimentos a serem realizados, consequentemente os maiores cuidados necessários, pode acarretar uma transição mais traumática quando comparada à de uma reabilitação com prótese total mediata 
(KRALJEVIE S, et al., 2001). Irregularidades no rebordo alveolar (BATES JF e STAFFORD GD, 1971) e tecidos moles, após as exodontias, levam à necessidade de reembasamentos periódicos (BATES JF e STAFFORD GD, 1971; ST GEORGE G, et al., 2010) e a necessidade de confecção de uma nova prótese, definitiva, após o período de completa cicatrização (PATEL J, et al., 2018), fato que aumenta o custo para o paciente (BATES JF e STAFFORD GD, 1971). Devido ao posicionamento favorável dos dentes presentes em nosso caso, não foi necessária a utilização de uma moldeira individual, simplificando as etapas clínicas prévias à cirurgia. A paciente em questão tinha presente somente um pré-molar (15) para a manutenção da DVO, sendo realizadas as exodontias dos elementos 16,18 e 28 previamente à realização da etapa de confecção da PTI, visando a facilitar a instalação, individualização das bases de prova e dos planos de orientação. (KRALJEVIE S, et al., 2001; SILVA RJ, et al., 2008; TELLES D, 2009; TONG DC, et al., 2014; GAVAZZONI A, et al., 2015)

$\mathrm{Na}$ paciente em questão, as exodontias dos remanescentes dentários, foram realizadas em uma etapa cirúrgica, na qual todos os elementos foram removidos com o auxílio de fórceps número 150, seguida de incisão interdental para ampliação da visualização do campo operatório, possibilitando a regularização do rebordo de maneira adequada. Durante a realização da regularização do rebordo alveolar, fez-se necessária a utilização de um guia cirúrgico, que neste caso, foi utilizada a própria prótese imediata, diminuindo, assim, o custo do procedimento laboratorial (KRALJEVIE S, et al., 2001; GAVAZZONI A, et al., 2015). Segundo SCHROPP et al., 2002; HAMMERLE et al., 2012; a reabsorção das cristas alveolares, que levam à redução na altura e na largura do rebordo, após a realização de exodontias, é um processo de remodelação óssea que leva a uma perda de, aproximadamente, dois terços do volume total após seis meses de cicatrização. Esse padrão de remodelação óssea é diferente para cada área. Na mandíbula anterior, observa-se uma reabsorção vertical e horizontal (ângulo nasolabial); na mandíbula posterior, ocorre uma reabsorção vertical (dimensão vertical), e, na maxila, tanto vertical como horizontal (dimensão vertical) (ST GEORGE G, et al., 2010).

A instalação da PTI será realizada após o momento cirúrgico, sendo importante orientar o paciente a não removê-la até a avaliação que deverá ser realizada após 24 horas, para minimizar o edema basal (ST GEORGE GS, et al., 2010; SADOWSKY SJ, et al., 2013; TELLES D, 2009). A remoção das suturas depende da avaliação no $7^{\circ}$ dia de pós-operatório, no qual serão realizados ajustes oclusais. SHIBAYAMA R et al. (2012) citam que se fazem necessárias várias sessões clínicas de controle da prótese total imediata, visto que ela perderá suporte, estabilidade e retenção, de acordo com a progressiva remodelação óssea. Nossa paciente foi orientada a não remover a prótese nas primeiras 24 horas, para permitir a estabilização do coágulo e a contenção do edema pós-operatório. Seu controle operatório foi realizado no $7^{\circ}, 21^{\circ}$ e $30^{\circ}$ dia pós-operatório, sendo, nesses primeiros, realizado reembasamentos com condicionador de tecido soft (Softconfort / Dencril Produtos Odontológicos). No controle de 30 dias, o reembasamento foi realizado com material rígido (Cold Liner Reembase / TDV Dental LTDA), trazendo como vantagens a melhor adesão à base da prótese, consequentemente, aumentando a sua durabilidade (GOIATO MC, et al., 2013).

A reabilitação estética e funcional do sorriso por meio das próteses totais imediatas, é uma modalidade de tratamento extremamente útil para a resolução dos casos onde os pacientes não queiram se sujeitar a um período de pós-operatório sem dentes. O uso destas próteses, permite uma melhor cicatrização óssea e tecidual, além de permitir a devolução imediata da estética. O prognóstico clínico deste tratamento, dependerá da proservação de cada caso, com visitas periódicas para ajustes, e da colaboração do paciente. Esses aspectos determinarão o sucesso e a longevidade do trabalho protético executado. Nesse contexto, o presente estudo discutiu os principais aspectos que são necessários para que uma reabilitação imediata por meio de prótese total possa ser executada.

\section{REFERÊNCIAS}

1. BATES JF, STAFFORD GD. Immediate complete dentures. British Dental Journal, 1971; 131(11):500-503.

2. BÜTTEL AE, et al. Immediate loading of two unsplinted mandibular implants in edentulous patients with an implantretained overdenture: an observational study over two years. Schweiz Monatsschr Zahnmed. 2012; $122(5)$ : $392-397$. 
3. DE MEDEIROS JJ, et al. Edentulismo, uso e necessidade de prótese e fatores associados em município do nordeste brasileiro. Pesquisa Brasileira em Odontopediatria e Clínica Integrada. 2012; 12(4): 573-578.

4. EMAMI E, et al. The impact of edentulism on oral and general health. International Journal of Dentistry. 2013; 2013: 1-7.

5. FELTON DA. Complete edentulism and comorbid diseases: an update. Journal of Prosthodontics. 2015; 25(1): 5-20.

6. GAVAZZONI A, et al. Immediate complete denture: a contemporary view. Journal of Research in Dentistry. 2015; 3(2): 646-653.

7. GEORGE GS, et al. Immediate dentures: 1. Treatment planning. Dental Update. 2010; 37(2): 82-91.

8. Goiato MC, et al. Técnicas de reembasamento para prótese total. Revista Odontológica de Araçatuba. 2013; 34(2): 61-66.

9. GOMES AC, et al. Reabilitação bucal com prótese total imediata. Full Dentistry in Science. 2014; 5(20): 590-594.

10. Hammerle $\mathrm{CH}$, et al. Evidence-based knowledge on the biology and treatment of extraction sockets. Clinical Oral Implants Research. 2012; 23(5): 80-82.

11. JABLONSKI RY, BARBER MW. Restorative dentistry for the older patient cohort. British Dental Journal. 2015; 218(6): 337-342.

12. JOGEZAI U, et al. Immediate dentures part 1: assessment and treatment planning. Dental Update. 2018; 45(7): 617624.

13. JOHNSON K. A study of the dimensional changes occurring in the maxilla following closed face immediate denture treatment. Australian Dental Journal. 1966; 14(6): 370-376.

14. KRALJEVIE S, et al. Immediate Complete Denture. Acta Stomatologica Croatica. $2001 ; 35(2): 281-285$.

15. MELETI VR, et al. Prótese total imediata: uma solução estética e funcional. Revista Odontológica do Brasil Central. 2002; $11(82): 50-53$.

16. PATEL J, et al. Complete dentures: an update on clinical assessment and management: part 1. British Dental Journal. 2018; 225: 707-714.

17. POLZER I, et al. The association of tooth loss with all-cause and circulatory mortality. Is there a benefit of replaced teeth? A systematic review and meta-analysis. Clinical Oral Investigation. 2012;16(2): 333-351.

18. PRESTON AJ. Dental management of the elderly patient. Dental Update. 2012; 39(2): 141-144.

19. SADOWSKY SJ, et al. A technique to correct incisal plane error in maxillary immediate denture therapy. Journal of Prosthetic Dentistry. 2013; 110: 141-143.

20. SCHROPP L, et al. Bone healing changes and soft tissue contour changes following single tooth extraction: a clinical and radiographic 12-month prospective study. International Journal of Periodontics and Restorative Dentistry. 2003; 23: 313-323.

21. SHIBAYAMA R, et al. Próteses totais imediatas convencionais. Revista Odontológica de Araçatuba. 2006; 27(1): 6772.

22. SILVA RJ, et al. Reembasamento direto para prótese total: uma alternativa simples e eficiente-relato de caso clínico. International Journal of Dentistry. 2008; 7(3): 190-194.

23. ST GEORGE G, et al. Immediate dentures: 1. Treatment planning. Dental Update. 2010; 37(2): 86-88.

24. TELLES D. Prótese Total - Convencional e Sobre Implantes. 1nd ed. São Paulo: Santos; 2009; 492p.

25. Tong DC, et al. Operative complications following dental extractions at The School of Dentistry. University of Otago. New Zealand Dental Journal. 2014; 110: 51-55.

26. ZARB GA, et al. Prosthodontic treatment for edentulous patients: complete dentures and implant-supported prostheses. 13nd ed. St. Louis: Mosby-Elsevier. 2013; 494p.

27. ZITZMANN NU, MARINELLO CP. A review of clinical and technical considerations for fixed and removable implant prostheses in the edentulous mandible. International Journal of Prosthodontics. 2002; 15(1): 65-72. 